\title{
Curcumin enhances temsirolimus-induced apoptosis in human renal carcinoma cells through upregulation of YAP/p53
}

\author{
SHAN XU ${ }^{1,2}$, ZHENG YANG $^{1,2}$, YIZENG FAN ${ }^{1,2}$, BING GUAN $^{1,2}$, JING JIA $^{1,2}$, YANG GAO $^{1,2}$,

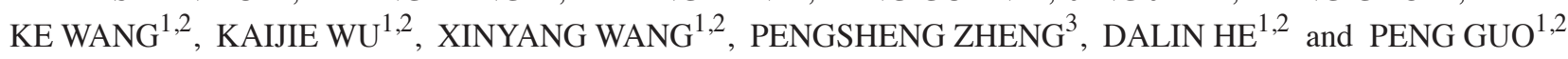 \\ ${ }^{1}$ Department of Urology, The First Affiliated Hospital of Xi'an Jiaotong University, Xi'an; ${ }^{2}$ Oncology Research Lab, \\ Key Laboratory of Environment and Genes Related to Diseases, Ministry of Education, Xi'an; \\ ${ }^{3}$ Department of Reproductive Medicine, The First Affiliated Hospital, Xi'an Jiaotong University Medical School, \\ Xi'an, Shaanxi 710061, P.R. China
}

Received July 21, 2015; Accepted October 4, 2016

DOI: $10.3892 / \mathrm{ol} .2016 .5376$

\begin{abstract}
Curcumin has frequently been used as a therapeutic agent in the treatment of various types of disease and is known to enhance the drug sensitivity of cells. In the present study, the combined effect of curcumin and temsirolimus treatment on apoptosis in human renal cell carcinoma (RCC) cells was investigated. Temsirolimus is an inhibitor of the mechanistic target of rapamycin signaling pathway and used in the first-line treatment of metastatic RCC. It was demonstrated that curcumin combined with temsirolimus markedly induced apoptosis in RCC cells, however this effect was not observed following curcumin or temsirolimus treatment alone. Co-treatment with temsirolimus and curcumin led to the activation of cleaved poly ADP-ribose polymerase and caspase 3, upregulation of p53 expression and nuclear translocation, and downregulation of B-cell lymphoma 2 protein expression. Furthermore, curcumin treatment was demonstrated to increase Yes-associated protein (YAP) expression in a time-dependent manner, which was concurrent with the curcumin-induced expression pattern of p53 after $2 \mathrm{~h}$. In addition, knockdown of YAP by small interfering RNA caused the attenuation of curcumin-induced increased p53 expression in RCC cells. In conclusion, the present results indicate that combined curcumin and temsirolimus treatment has a synergistic effect on apoptosis in human RCC cells, through the activation of p53. Mechanistically, YAP is essential in the induction of p53 expression by curcumin. Furthermore, the results suggest that pre-treatment or co-treatment of cells with low concentration
\end{abstract}

Correspondence to: Professor Dalin He or Professor Peng Guo, Department of Urology, The First Affiliated Hospital of Xi'an Jiaotong University, 277 Western Yanta Road, Xi'an, Shaanxi 710061, P.R. China

E-mail: dalinhexjtu@163.com

E-mail: guopeng661@mail.xjtu.edu.cn

Key words: renal cell carcinoma, Yes-associated protein, p53, curcumin, temsirolimus curcumin enhances the response to targeted drugs, and this presents a potentially novel and efficient strategy to overcome drug resistance in human RCC.

\section{Introduction}

According to the 2014 World Cancer Report, kidney cancer was the sixteenth most common cause of cancer-associated mortality worldwide in 2012 (1). Although targeted drug therapy for renal cell carcinoma (RCC) has been improved, the response rates of targeted drugs in metastatic RCC (mRCC) remain poor, and resistance to chemotherapy is a primary obstacle in RCC treatment $(1,2)$.

Mechanistic target of rapamycin (mTOR) signaling is highly induced in almost all types of cancer cell and is important in cell proliferation, metabolism and survival. Temsirolimus is an mTOR inhibitor used in the first-line chemotherapeutic treatment of mRCC; however, the majority of patients acquire temsirolimus resistance during the course of treatment $(3,4)$. Therefore, novel therapeutic strategies to overcome drug resistance, improve clinical effects and decrease toxicity are required.

Curcumin, a polyphenolic phytochemical derived from the rhizomes of the Curcuma longa plant, is one of the best-studied plant derivatives in the world $(5,6)$. Curcumin has been used as a therapeutic agent in the treatment of various types of disease due to its apoptotic inductive, chemopreventive, anti-angiogenic and anti-invasive/metastatic properties (7). Curcumin may induce apoptosis through the reshaping of multiple molecular targets, including the upregulation of death receptor $4 / 5$ expression, activation of caspase-3, release of cytochrome $c$, inhibition of B-cell lymphoma (Bcl)-extra large protein, $\mathrm{Bcl}-2$ protein and c-Myc mRNA expression, and activation of the mitochondrial signaling pathway $(5,8-10)$. Furthermore, curcumin has been reported to increase radio- and chemosensitivity $(11,12)$. Seo et al (12) reported that combined curcumin and NVP-BEZ235 treatment had a synergistic effect on apoptosis through the inhibition of Bcl-2 expression in a p53-dependent manner, however the underlying mechanism remains unclear.

Previously, it has been observed that curcumin is able to regulate the expression of YAP in bladder cancer cells (6). 
Therefore, in the present study, the combined effect of curcumin and temsirolimus treatment on apoptosis in RCC cells was investigated, and it was determined whether the enhanced inhibitory effect of temsirolimus was caused by curcumin-mediated Yes-associated protein (YAP)/p53 induction.

\section{Materials and methods}

Cell culture and temsirolimus/curcumin treatment. Human RCC cell lines Caki-1 and OS-RC-2, purchased from American Type Culture Collection (Manassas, VA, USA), were maintained in RPMI-1640 (Gibco; Thermo Fisher Scientific, Inc., Waltham, MA, USA) containing 10\% (v/v) fetal bovine serum (FBS; Hyclone; GE Healthcare Life Sciences, Logan, UT, USA) at $37^{\circ} \mathrm{C}$ in a humidified $5 \% \mathrm{CO}_{2}$ incubator. Caki-1 cells were treated with final concentrations of $10 \mu \mathrm{M}$ temsirolimus alone, $15 \mu \mathrm{M}$ curcumin alone or $10 \mu \mathrm{M}$ temsirolimus and $15 \mu \mathrm{M}$ curcuma and incubated at $37^{\circ} \mathrm{C}$ for $48 \mathrm{~h}$; cells were treated with dimethyl sulfoxide (DMSO) as a control. OS-RC-2 cells were treated with final concentrations of $15 \mu \mathrm{M}$ temsirolimus alone, $10 \mu \mathrm{M}$ curcumin alone or $15 \mu \mathrm{M}$ temsirolimus and $10 \mu \mathrm{M}$ curcumin, or DMSO as a control, and subsequently incubated at $37^{\circ} \mathrm{C}$ for $48 \mathrm{~h}$.

Cell flow cytometric analysis. Human RCC cell lines Caki-1 and OS-RC-2 were cultured in RPMI-1640 medium supplemented with $10 \%$ FBS in $6 \mathrm{~cm}$-dishes. Prior to treatment, cells were provided with fresh media and subsequently incubated with the aforementioned concentrations of temsirolimus, curcumin and temsirolimus combined with curcumin, for $48 \mathrm{~h}$. The cells were resuspended and washed with $500 \mathrm{ml}$ phosphate-buffered saline (PBS), and incubated with Annexin-V-Fluorescein (Roche Applied Science, Penzberg, Germany) in a 4-(2-hydroxyethyl)-1-piperazineethanesulfonic acid buffer containing propidium iodide at room temperature for $20 \mathrm{~min}$. The stained cells were analyzed by fluorescence activated cell sorting using a FACSCalibur ${ }^{\mathrm{TM}}$ flow cytometer (BD Biosciences, Franklin Lakes, NJ, USA).

TUNEL analysis. Cells cultured in a Millicell ${ }^{\circledR}$ EZ SLIDE 8-well glass (Merck Millipore, Darmstadt, Germany) were washed with PBS three times, fixed with 4\% paraformaldehyde for 30 min, washed with PBS again and treated with permeabilization solution (1\% Triton X-100 ${ }^{\mathrm{TM}}$ (Sigma-Aldrich; Merck Millipore) in PBS) for $5 \mathrm{~min}$. Subsequently, incubated with terminal deoxynucleotidyl transferase-containing reaction mixture, which was part of the One Step TUNEL Apoptosis Assay kit (Beyotime Institute of Biotechnology, Shanghai, China), for $60 \mathrm{~min}$ at $37^{\circ} \mathrm{C}$ in the dark. Cells were washed with PBS three times and stained with streptavidin-tetramethylrhodamine for $30 \mathrm{~min}$ at $37^{\circ} \mathrm{C}$ in the dark. Subsequently, cells were washed with PBS three times and stained with 4',6-diamidino-2-phenylindole (DAPI) for $10 \mathrm{~min}$ in the dark. Finally, the samples were visualized using a confocal laser scanning microscope (Nikon A1R/A1; Nikon Corporation, Tokyo, Japan).

Western blot analysis. Total cellular protein was extracted from cells lysed in radioimmunoprecipitation assay buffer
[50 mM Tris (pH 8.0), $150 \mathrm{mM} \mathrm{NaCl}, 0.1 \%$ sodium dodecyl sulfate (SDS), $1 \%$ NP-40 and $0.5 \%$ sodium deoxycholate] containing protease inhibitors: $1 \%$ protease inhibitor cocktail and $1 \mathrm{mM}$ phenylmethanesulfonyl fluoride (Sigma-Aldrich; Merck Millipore). The concentration of protein was detected by Bradford assay protein quantitation kit (Abcam, Cambridge, UK). A total of $30 \mu \mathrm{g}$ protein was separated by $12 \%$ SDS-polyacrylamide gel electrophoresis and transferred onto nitrocellulose membranes. The membranes were blocked with 5\% skimmed milk reconstituted in Tris-buffered saline with $0.1 \%$ Tween $20(\mathrm{pH} \mathrm{7.6)}$ at room temperature for $1 \mathrm{~h}$, and washed with PBS three times, followed by incubation at $4^{\circ} \mathrm{C}$ overnight with primary antibody. Primary antibodies used were as follows: Poly ADP-ribose polymerase (PARP) (dilution 1:1,000; \#9532), caspase 3 (dilution, 1:1,000; \#9662), YAP (dilution, 1:1,000; \#14074; All Cell Signaling Technology, Inc., Danvers, MA, USA), p53 (dilution, \#sc-47698 1:400; Santa Cruz Biotechnology, Inc., Dallas, TX, USA) and glyceraldehyde-3-phosphate dehydrogenase (GAPDH; dilution 1:10,000; \#KC-5G4; KangChen Biotech, Shanghai, China). Following incubation with the primary antibodies, membranes were incubated with secondary antibody [peroxidase-conjugated affinipure goat anti-Rabbit IgG (\#ZB-2301; dilution, 1:2,000; Beijing Zhongshan Golden Bridge Biotechnology, Co., Ltd., Beijing, China) and peroxidase-conjugated affinipure goat anti-Mouse IgG (\#ZB-2305; dilution, 1:2,000; Beijing Zhongshan Golden Bridge Biotechnology, Co., Ltd.)] for $1 \mathrm{~h}$ at room temperature. Immunoreactive signals were detected using a WesternBright Quantum HRP substrate kit (Advansta, Inc., Menlo Park, CA, USA), visualized by a Molecular Imager ChemiDoc XRS system (Bio-Rad Laboratories, Inc., Hercules, CA, USA). Immunoblotting for GAPDH was performed as an internal control.

Immunofluorescence assay. Cells were cultured in a Millicell EZ SLIDE 8-well glass and washed with PBS three times, prior to being fixed in $4 \%$ paraformaldehyde for $30 \mathrm{~min}$. Cells were subsequently washed with PBS again, treated with permeabilization solution (1\% Triton X-100 in PBS), washed with PBS three times, and blocked with $1 \%$ bovine serum albumin (BSA; Sigma-Aldrich; Merck Millipore) in PBS for $1 \mathrm{~h}$. Samples were subsequently incubated with anti-p53 primary antibody in antibody dilution buffer (1\% BSA in 1xPBS) overnight at $4^{\circ} \mathrm{C}$. Samples were washed with PBS three times and incubated with secondary antibody tetramethylrhodamine-conjugated affinipure goat anti-Mouse IgG (\#ZF-0313; dilution, 1:200; Beijing Zhongshan Golden Bridge Biotechnology, Co., Ltd.) and fluorescein isothiocyanate-conjugated affinipure goat anti-Rabbit IgG (\#ZF-0315; dilution, 1:200; Beijing Zhongshan Golden Bridge Biotechnology, Co., Ltd.) for $60 \mathrm{~min}$ at room temperature, followed by DAPI staining $(1: 5,000)$ for $5 \mathrm{~min}$ in the dark. Samples were examined using laser scanning confocal microscopy (Nikon A1R/A1).

Small interfering RNA (siRNA) transfection. The YAP-siRNA (siYAP) and non-specific control (NC) siRNA (siNC) used in the present study were purchased from Guangzhou RiboBio Co., Ltd. (Guangzhou, China). The sequence of siYAP was: 5'-GCGTAGCCAGTTACCAACA-3' and the sequence of siNC was: 5'-UUCUCCGAACGUGUCACGUTT-3'. Cells were 
Table I. Primer sequences used in reverse transcription-quantitative polymerase chain reaction.

\begin{tabular}{lll}
\hline Gene & \multicolumn{1}{c}{ Forward primer } & Reverse primer \\
\hline GAPDH & ATGGGGAAGGTGAAGGTCGG & GACGGTGCCATGGAATTTGC \\
YAP & AATTTGCCCAGTTATACCTCAGTG & CACATCAAGGCTATGATTCAAACTC \\
\hline
\end{tabular}

GAPDH, glyceraldehyde 3-phosphate dehydrogenase; YAP, Yes-associated protein.

transfected with siRNA using the X-tremeGENE ${ }^{\mathrm{TM}}$ siRNA Transfection Reagent (Roche Applied Science) according to the manufacturer's protocol.

Reverse transcription-quantitative polymerase chain reaction (RT-qPCR). Total RNA was isolated using the RNA fast $200 \mathrm{kit}$ (Feijie Biotech, Shanghai, China) and reverse-transcribed into cDNA using the PrimeScript RT-PCR kit (Takara Biotechnology Co., Ltd., Dalian, China). Relative gene expression was studied using the Real-Time PCR Detection system (Bio-Rad Laboratories, Inc.) and SYBR Green PCR Master mix (Takara Biotechnology Co., Ltd.). The thermocycling conditions were as follows: $95^{\circ} \mathrm{C}$ for $30 \mathrm{sec}$, followed by 40 cycles at $95^{\circ} \mathrm{C}$ for $5 \mathrm{sec}$, and $60^{\circ} \mathrm{C}$ for $30 \mathrm{sec}$. Primer sequences used are presented in Table I. Transcriptional expression of specific genes was calculated using the $2^{-\Delta \Delta \mathrm{Cq}}$ method (13). GAPDH was used for normalization.

Statistical analysis. Results are presented as the mean \pm standard error of triplicate experiments. Differences between two groups were compared by the Student's $t$-test. All data analyses were performed using GraphPad Prism version 6 software (GraphPad Software, Inc., La Jolla, CA, USA). P<0.05 was considered to indicate a statistically significant difference.

\section{Results}

Synergistic effect of temsirolimus and curcumin treatment on apoptosis. To investigate whether combined treatment with curcumin and temsirolimus could induce cell death in RCC cells, the Caki-1 and OS-RC-2 renal cancer cells were treated with these two agents. Apoptosis was detected using flow cytometry and the TUNEL assay. As demonstrated in Fig. 1A, Caki-1 and OS-RC-2 cells treated with temsirolimus or curcumin alone did not exhibit increased apoptosis, however combined curcumin and temsirolimus treatment did cause increased apoptosis in RCC cells. The percentage of apoptotic Caki-1 cells following combined treatment was $52.90 \%$, which was significantly increased compared with temsirolimus $(6.78 \%)$ and curcumin $(8.38 \%)$ treatment alone (Fig. 1B; $\mathrm{P}=0.013$ and $\mathrm{P}=0.009$, respectively). The percentage of apoptotic OS-RC-2 cells following combined treatment was $41.82 \%$, which was significantly increased compared with temsirolimus $(3.05 \%)$ and curcumin $(2.52 \%)$ treatment alone (Fig. $1 \mathrm{C} ; \mathrm{P}=0.005$ and $\mathrm{P}=0.003$, respectively).

Furthermore, the TUNEL assay was used to detect DNA fragmentation following the treatment of RCC cells with temsirolimus/curcumin alone or in combination. As shown in Fig. 1D and E, treatment with temsirolimus or curcumin alone did not induce DNA fragmentation in the RCC cell lines, however co-treatment increased DNA fragmentation in Caki-1 (Fig. 1D) and OS-RC-2 (Fig. 1E) cells. The results of the present study indicate that temsirolimus and curcumin have a synergistic effect in inducing apoptosis in RCC cells.

Effect of combined treatment with temsirolimus and curcumin on apoptosis-associated proteins. In order to elucidate the mechanism of combined temsirolimus/curcumin-mediated apoptosis in human RCC cells, western blot analysis was performed to detect the expression of caspase 3 and PARP, which serve important roles in apoptotic progression. Pro-caspase 3 levels were significantly downregulated in Caki-1 cells treated with combined temsirolimus and curcumin, compared with Caki-1 cells treated with temsirolimus or curcumin alone (Fig. 2A; $\mathrm{P}<0.05$ vs. control). Similarly, pro-caspase 3 levels were significantly downregulated in OS-RC-2 cells treated with combined temsirolimus and curcumin compared with OS-RC-2 cells treated with temsirolimus or curcumin alone (Fig. 2B; $\mathrm{P}=0.025$ and $\mathrm{P}=0.039$, respectively). Furthermore, cleaved caspase 3 (17 and $19 \mathrm{kDa}$ ) was observed only in the combined treatment groups. In addition, cleaved PARP, an index of caspase 3, was only observed in the combined treatment groups. The results of the present study indicate that combined temsirolimus and curcumin treatment induces apoptosis in a caspase-dependent manner.

Combined treatment with temsirolimus and curcumin induces apoptosis through altered p53 expression and distribution. Cellular p53 is activated as a result of stress, including DNA damage (14), and is an essential regulator of apoptosis (15). Therefore, the effect of curcumin and temsirolimus treatment on p53 expression was analyzed. p53 expression was markedly upregulated in RCC cells treated with curcumin alone between 4 and $24 \mathrm{~h}$, while in the combined treatment groups, p53 protein expression was only upregulated between 4 and $12 \mathrm{~h}$, and decreased at $24 \mathrm{~h}$ (Fig. 3A). No changes in p53 expression were observed in the DMSO or temsirolimus alone treatment groups (Fig. 3A).

The effect of curcumin on p53 expression and distribution was further examined using an immunofluorescence assay. As shown in Fig. 3B, p53 was translocated into the nucleus following $6 \mathrm{~h}$ treatment with curcumin alone or in combination with temsirolimus, however this phenomenon was not observed in cells treated with temsirolimus alone or in the control group. The results of the immunofluorescence assay suggest that p53 is activated by curcumin and explain how combined treatment induces apoptosis. The results of the present study indicate that combined treatment with temsirolimus and 
A

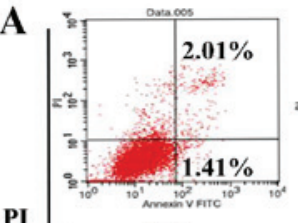

PI

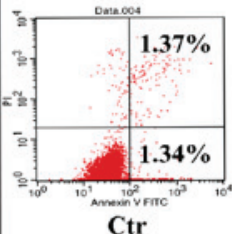

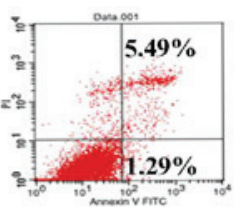
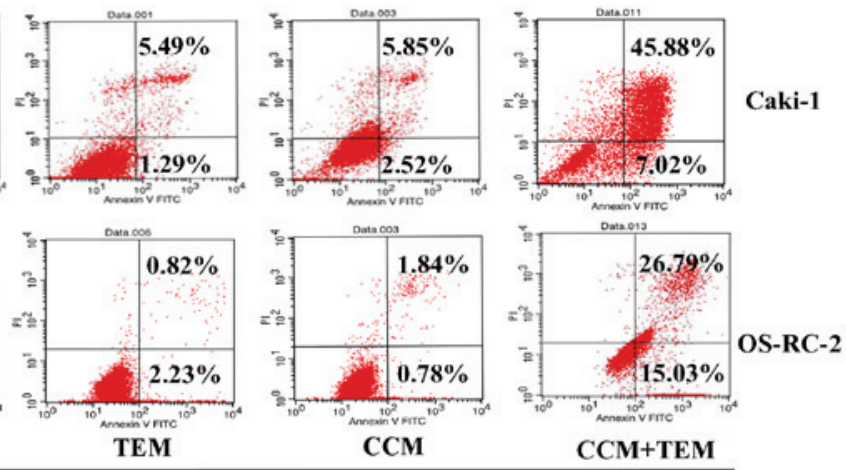

AnnexinV

B

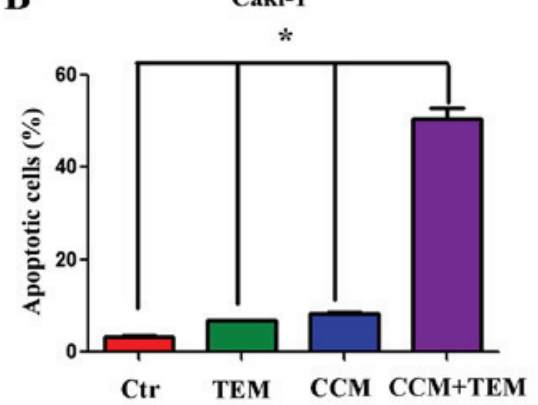

D

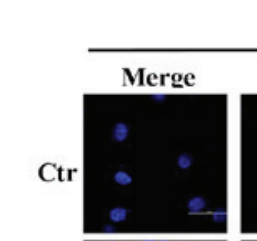

Caki-1
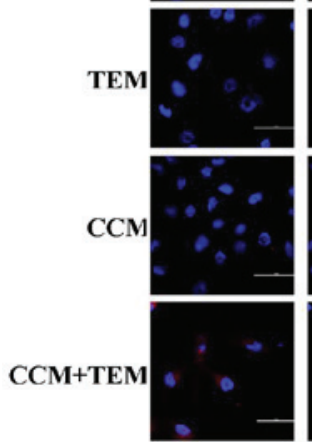

DAPI dUTP-TRITC
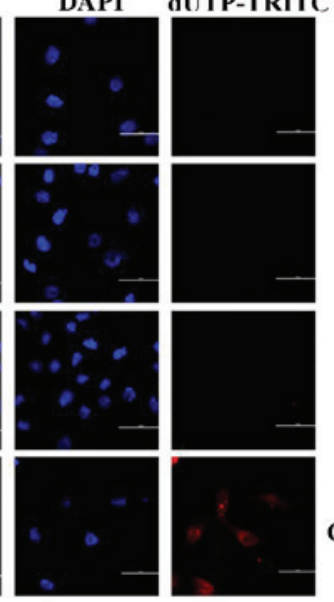

C

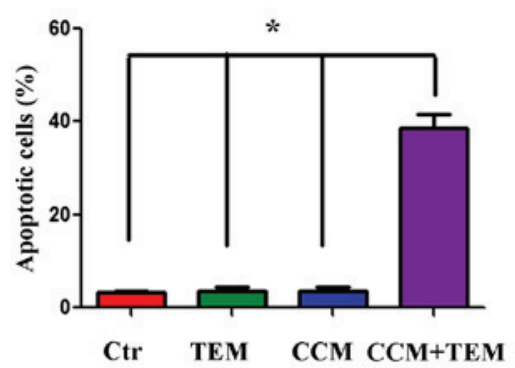

$\mathbf{E}$

OS-RC-2
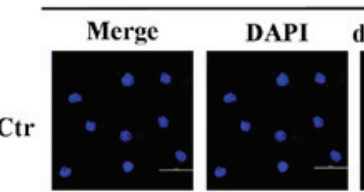

dUTP-TRITC

ctr
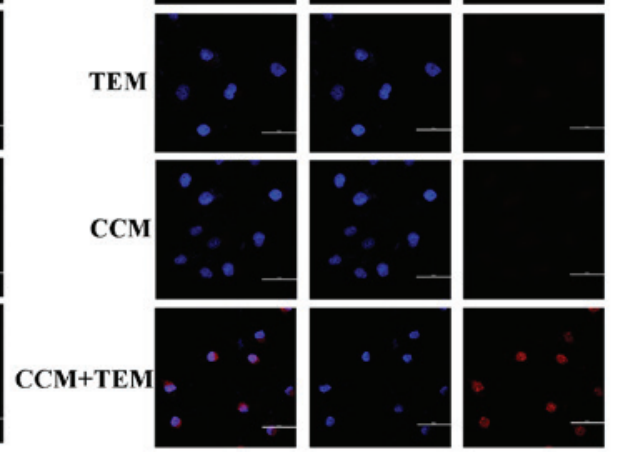

Figure 1. Synergistic effect of treatment with TEM and CCM on apoptosis in renal cell carcinoma cell lines. (A) Caki-1 and OS-RC-2 cells were treated with TEM alone, CCM alone, TEM and CCM, or the Ctr for $48 \mathrm{~h}$. Cells were stained using Annexin-V-Fluorescein and PI at room temperature for 20 min, and apoptosis was measured using flow cytometry. The percentage of apoptotic cells in the (B) Caki-1 and (C) OS-RC-2 cell lines was calculated and analyzed using GraphPad Prism software. Values are presented as the mean \pm standard deviation from three independent samples ( $\mathrm{P}<0.05$ vs. Ctr). Following treatment with TEM alone, CCM alone, TEM and CCM, or the Ctr for 48 h, DNA strand breakage was detected using the terminal deoxynucleotidyl transferase-mediated dUTP Nick-End Labeling assay and observed using a confocal laser scanning microscope in (D) Caki-1 cells and (E) OS-RC-2 cells. TEM, temsirolimus; CCM, curcumin; Ctr, control; PI, propidium iodide; dUTP-TRITC, 2'-deoxyuridine, 5'-triphosphate-tetramethylrhodamine; DAPI, 4',6-diamidino-2-phenylindole.

curcumin increases apoptosis, which is at least partly due to curcumin-mediated p53 induction. However, the underlying mechanism of curcumin-mediated induced p53 expression remains unclear.

Curcumin-induced expression of $Y A P / p 53$. YAP is the major downstream effector of the Hippo signaling pathway and is able to directly regulate the transcription of p53 (16). Previously, it was demonstrated that curcumin is able to regulate the expression of YAP in bladder cancer cells (6), therefore curcumin may regulate the expression of YAP in RCC cells.
To investigate changes in YAP expression following treatment with temsirolimus plus curcumin, YAP expression was detected by western blot analysis at the following time points: $0,1,2,4,8,12$ and $24 \mathrm{~h}$. As shown in Fig. 4A, YAP protein expression increased between 2 and $12 \mathrm{~h}$ in a time-dependent manner and subsequently decreased at $24 \mathrm{~h}$, which is concurrent with the expression pattern of p53 following treatment with curcumin alone. However, the induction of p53 was $\sim 2 \mathrm{~h}$ behind the induction of YAP. The results of the present study suggest that expression of YAP and p53 is increased in RCC cells following treatment with curcumin. 
A
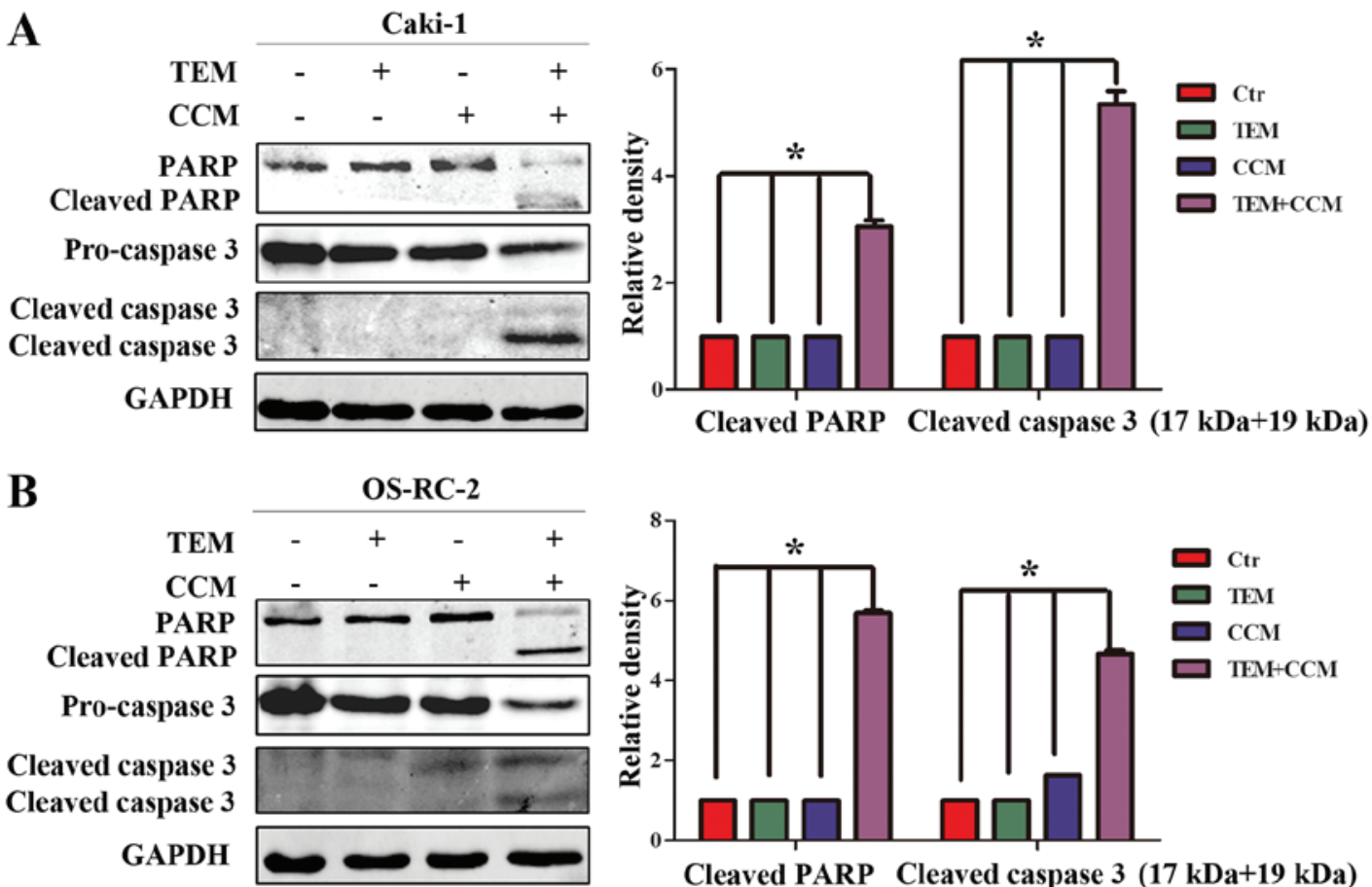

Figure 2. Effect of combined treatment with TEM and CCM on apoptotic proteins. (A) Caki-1 and (B) OS-RC-2 cells were treated with TEM alone, CCM alone, TEM and CCM, or the Ctr for $48 \mathrm{~h}$. PARP and caspase 3 protein expression levels were determined in the two cell lines using western blot analysis ("P $<0.05$ vs. Ctr). TEM, temsirolimus; CCM, curcumin; Ctr, control; PARP, poly ADP-ribose polymerase; GAPDH, glyceraldehyde 3-phosphate dehydrogenase.

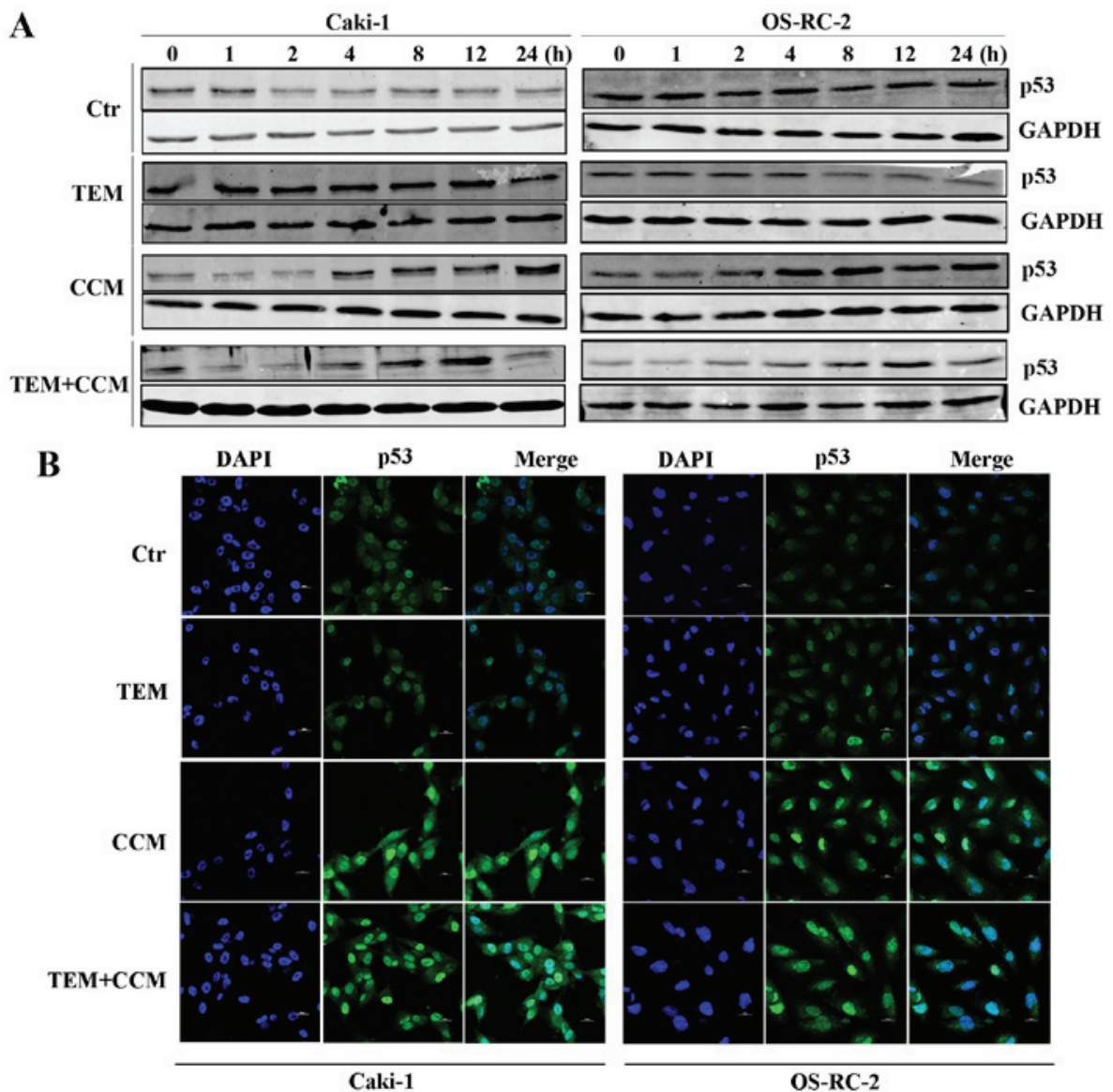

Figure 3. Combined treatment with TEM and CCM induces apoptosis through alterations in the expression and distribution profile of p53. (A) Caki-1 and OS-RC-2 cells were treated with TEM alone, CCM alone, TEM and CEM, or the Ctr, and p53 expression levels were determined using western blot analysis at various time points. (B) Caki-1 and OS-RC-2 cells were treated with TEM alone, CEM alone, TEM and CEM, or the Ctr for $6 \mathrm{~h}$. p53 expression and distribution was detected using immunofluorescence. TEM, temsirolimus; CCM, curcumin; Ctr, control; GAPDH, glyceraldehyde 3-phosphate dehydrogenase; DAPI, 4',6-diamidino-2-phenylindole. 
$\mathbf{A}$

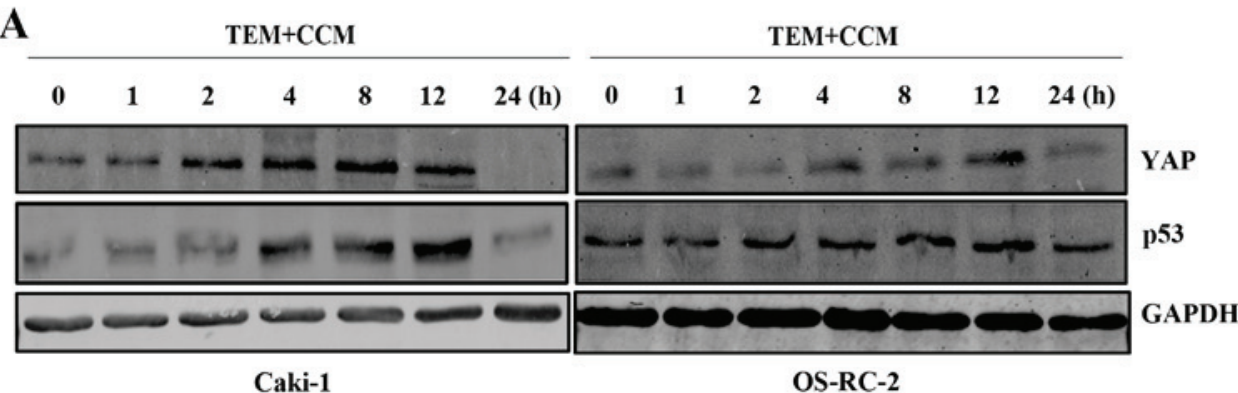

\section{B}

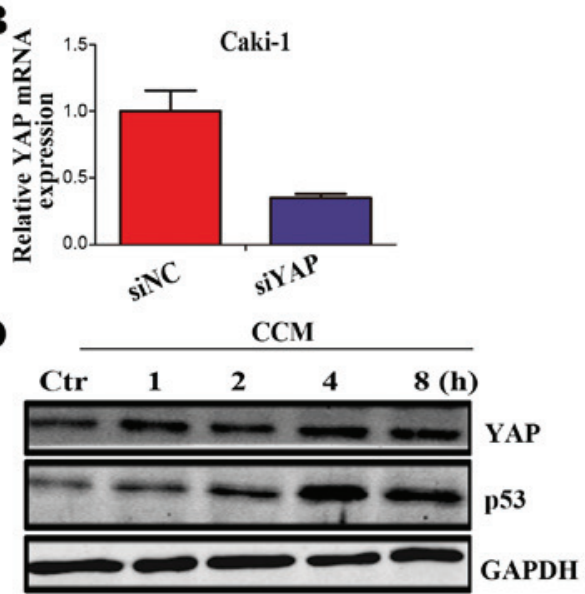

Caki-1/siNC
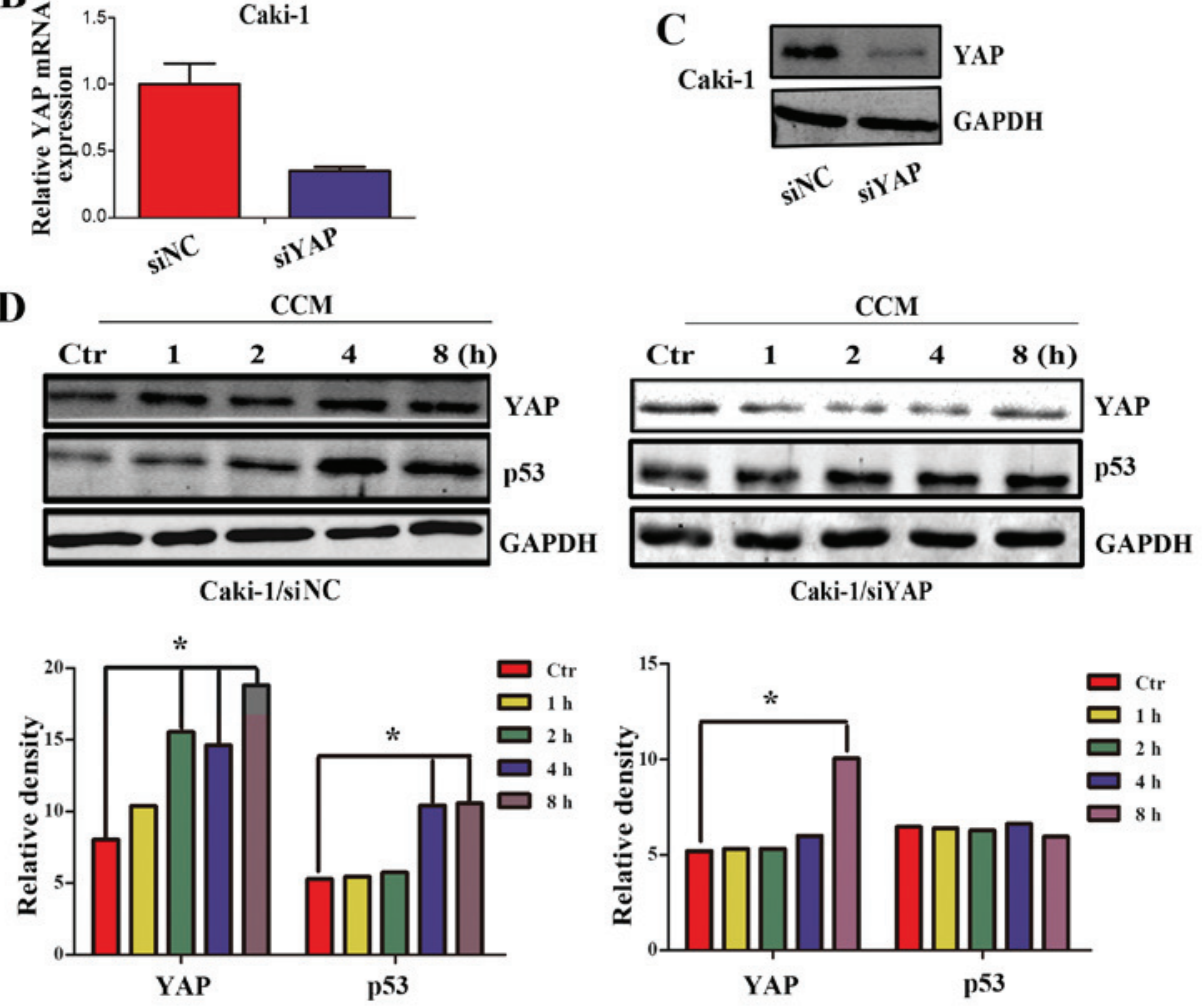

$\mathbf{E}$

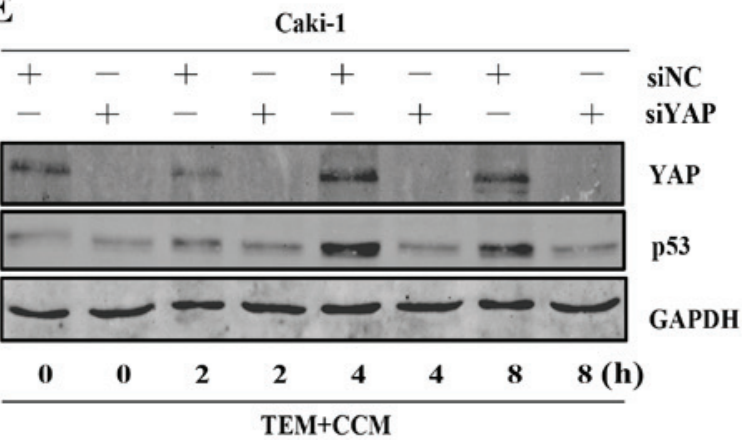

Figure 4. YAP is necessary in the activation of p53. (A) Caki-1 and OS-RC-2 cells were treated with TEM and CCM. YAP and p53 expression levels were determined using western blot analysis at various time points. Caki-1 cells were transfected with siYAP or siNC. A total of $48 \mathrm{~h}$ following transfection, cells were lysed and the expression of YAP was analyzed by (B) reverse transcription-quantitative polymerase chain reaction and (C) western blot analysis. (D) A total of $48 \mathrm{~h}$ following transfection, cells were treated with $10 \mu \mathrm{m} / \mathrm{ml} \mathrm{CCM}$, and YAP and p53 expression levels were subsequently analyzed at various time points by western blot analysis. (E) Caki-1/siNC and Caki-1/siYAP cells were treated with $10 \mu \mathrm{m} / \mathrm{ml} \mathrm{CCM}$ and $15 \mu \mathrm{m} / \mathrm{ml} \mathrm{TEM}$, and YAP and p53 expression levels were subsequently analyzed at various time points using western blot analysis ( $\mathrm{P}<0.05 \mathrm{vs}$. Ctr). YAP, Yes-associated protein; TEM, temsirolimus; $\mathrm{CCM}$, curcumin; si, small interfering; NC, non-specific control; Ctr, control; GAPDH, glyceraldehyde 3-phosphate dehydrogenase.

As YAP and p53 are activated by combined treatment with temsirolimus and curcumin, and YAP has an important function as an activator of p53 (16), it was further investigated whether curcumin activated 553 through YAP. Following transfection with YAP siRNA for $48 \mathrm{~h}$, Caki-1 cells exhibited a decrease in YAP mRNA and protein expression, when compared with the negative control cells (Fig. 4B and C). Caki-1/siNC and Caki-1/siYAP cells were subsequently treated with $10 \mu \mathrm{M} / \mathrm{ml}$ curcumin, and YAP and p53 protein levels were measured at various time points using western blot analysis. As shown in 
Fig. 4D, p53 expression in Caki-1/si YAP was not increased following treatment with temsirolimus plus curcumin for $8 \mathrm{~h}$, compared with the control cells. As shown in Fig. 4E, increased p53 protein expression was not induced in Caki-1/siYAP cells $8 \mathrm{~h}$ following treatment with curcumin, however significantly increased p53 expression was induced in Caki-1/siNC cells $4 \mathrm{~h}$ following treatment with curcumin $(\mathrm{P}<0.05$ vs. control). Furthermore, Caki-1 cells were treated with temsirolimus plus curcumin following transfection with siRNA for $48 \mathrm{~h}$. The results of the present study reveal that activation of p53 by curcumin in RCC cells is dependent on YAP.

\section{Discussion}

Deregulated apoptosis is a hallmark of cancer (15). p53 is reported to be a tumor suppressor protein involved in DNA repair, cell cycle arrest, senescence and apoptosis $(15,17)$. In the present study, Caki-1 and OS-RC-2 RCC cell lines, which contain wild type p53, were used as a cell model (18). The present results demonstrated that the expression of p53 is increased following combined treatment with temsirolimus and curcumin in Caki-1 and OS-RC-2 cells for 48 h. However, this effect is not observed following treatment with temsirolimus or curcumin alone. In addition, cellular p21 expression is increased following treatment with curcumin (data not shown). Previous research has demonstrated that when p53 is induced as a result of limited cell damage it activates growth-inhibitory genes to cause transient cell cycle arrest. However, p53 triggers the activation of apoptosis-associated genes when cells are exposed to extensive and irreparable damage $(15,19,20)$. Cells sustained mild damage following treatment with low concentration curcumin, leading to p53-mediated activation of p21 expression and subsequent cell cycle arrest. However, combined treatment with temsirolimus and curcumin caused severe damage to cells and this led to apoptosis.

YAP, the effector of the Hippo signaling pathway, is reported to be an oncoprotein involved in tumor progression, metastasis, proliferation, transformation, migration and invasion in RCC (21-23). However, the results of the present study suggest that YAP enhances the chemosensitivity of RCC cells by directly activating p53 expression. It was demonstrated that YAP protein was immediately increased following treatment with curcumin. Furthermore, following transfection with YAP siRNA, it was observed that YAP is essential for p53 activation. The results of the present study indicate that YAP functions as an apoptotic enhancer of chemotherapeutics in RCC and this is consistent with observations in other carcinoma cells (16). Chemoresistance in cells appears to be regulated by proliferative and anti-apoptotic signals, as well as metabolic pathways and changes in drug treatment. YAP may not only be regulated by the Hippo signaling pathway.

Curcumin is used widely in combinatory cancer therapy due to its anti-inflammatory and anticarcinogenic properties, as well as its low toxicity (8). The results of the present study demonstrated that curcumin combined with temsirolimus induces apoptosis. This is consistent with a previous study, in which combined treatment with curcumin and an mTOR inhibitor was demonstrated to induce apoptosis through p53-mediated downregulation of Bcl-2 pathway (12). However, the mechanism of altered p53 expression remained unclear.
In the present study, it was observed that treatment with low concentration curcumin activates the expression of YAP and p53 in a time-dependent manner but does not induce apoptosis. However combined treatment with low concentration curcumin and temsirolimus significantly induces apoptosis. Notably, treatment with high concentration curcumin alone induced apoptosis in Caki-1 and OS-RC-2 cell lines (data not shown). The molecular mechanism may be that low concentration curcumin activates YAP expression, which induces peak p53 expression and translocation to the nucleus. A second drug or stress may subsequently utilize p53 to induce the expression of apoptosis-associated genes, leading to apoptosis. The results demonstrate that YAP is a novel candidate target for curcumin-activated apoptosis.

In conclusion, the results of the present study demonstrate that co-treatment with temsirolimus and curcumin induces apoptosis in RCC cells through an increase in YAP/p53 expression. However, treatment with temsirolimus or curcumin alone does not induce apoptosis-associated gene expression. Furthermore, the molecular mechanism of temsirolimus and curcumin-induced apoptosis is the upregulation of YAP protein expression, and subsequently the upregulation of p53 expression. The present results suggest that combined treatment with curcumin and anti-cancer drugs has a synergistic effect in RCC cells.

\section{Acknowledgements}

The present study was supported by the National Natural Science Foundation of China (grant nos., 81602244, 81372279, and 81171953).

\section{References}

1. Frew IJ and Moch H: A clearer view of the molecular complexity of clear cell renal cell carcinoma. Annu Rev Pathol 10: 263-289, 2015.

2. Axelson H and Johansson ME: Renal stem cells and their implications for kidney cancer. Semin Cancer Biol 23: 56-61, 2013.

3. Kapoor A and Figlin RA: Targeted inhibition of mammalian target of rapamycin for the treatment of advanced renal cell carcinoma. Cancer 115: 3618-3630, 2009.

4. Gerullis H, Ecke TH, Eimer C, Heuck CJ and Otto T: mTOR-inhibition in metastatic renal cell carcinoma. Focus on temsirolimus: A review. Minerva Urol Nefrol 62: 411-423, 2010.

5. Tuorkey MJ: Curcumin a potent cancer preventive agent: Mechanisms of cancer cell killing. Interv Med Appl Sci 6: 139-146, 2014.

6. Gao Y, Shi Q, Xu S, Du C, Liang L, Wu K, Wang K, Wang X, Chang LS, He D and Guo P: Curcumin promotes KLF5 proteasome degradation through downregulating YAP/TAZ in bladder cancer cells. Int J Mol Sci 15: 15173-15187, 2014.

7. Rahmani AH, AlZohairy MA, Aly SM and Khan MA: Curcumin: A potential candidate in prevention of cancer via modulation of molecular pathways. Biomed Res Int 2014: 761608, 2014.

8. Troselj KG and Kujundzic RN: Curcumin in combined cancer therapy. Curr Pharm Des 20: 6682-6696, 2014.

9. Rana C, Piplani H, Vaish V, Nehru B and Sanyal SN: Downregulation of PI3-K/Akt/PTEN pathway and activation of mitochondrial intrinsic apoptosis by Diclofenac and Curcumin in colon cancer. Mol Cell Biochem 402: 225-241, 2015.

10. Rashid K and Sil PC: Curcumin enhances recovery of pancreatic islets from cellular stress induced inflammation and apoptosis in diabetic rats. Toxicol Appl Pharmacol 282: 297-310, 2015.

11. Toden S, Okugawa Y, Jascur T, Wodarz D, Komarova NL, Buhrmann C, Shakibaei M, Boland CR and Goel A: Curcumin mediates chemosensitization to 5-fluorouracil through miRNA-induced suppression of epithelial-to-mesenchymal transition in chemoresistant colorectal cancer. Carcinogenesis 36 : 355-367, 2015. 
12. Seo BR, Min KJ, Cho IJ, Kim SC and Kwon TK: Curcumin significantly enhances dual PI3K/Akt and mTOR inhibitor NVP-BEZ235-induced apoptosis in human renal carcinoma Caki cells through down-regulation of p53-dependent Bcl-2 expression and inhibition of Mcl-1 protein stability. PLoS One 9: e95588, 2014.

13. Livak KJ and Schmittgen TD: Analysis of relative gene expression data using real-time quantitative PCR and the 2(-Delta Delta C(T)) method. Methods 25: 402-408, 2001.

14. El-Deiry WS: The role of $\mathrm{p} 53$ in chemosensitivity and radiosensitivity. Oncogene 22: 7486-7495, 2003.

15. Aylon Y and Oren M: Living with p53, dying of p53. Cell 130: 597-600. 2007

16. Bai N, Zhang C, Liang N, Zhang Z, Chang A, Yin J, Li Z, Luo N, Tan X, Luo N, et al: Yes-associated protein (YAP) increases chemosensitivity of hepatocellular carcinoma cells by modulation of p53. Cancer Biol Ther 14: 511-520, 2013.
17. Vousden $\mathrm{KH}$ and Prives C: Blinded by the light: The growing complexity of p53. Cell 137: 413-431, 2009.

18. Zhou X, Tolstov Y, Arslan A, Roth W, Grüllich C, Pahernik S, Hohenfellner $M$ and Duensing S: Harnessing the p53-PUMA Axis to overcome DNA damage resistance in renal cell carcinoma. Neoplasia 16: 1028-1035, 2014.

19. Harris SL and Levine AJ: The p53 pathway: Positive and negative feedback loops. Oncogene 24: 2899-2908, 2005

20. Laptenko $\mathrm{O}$ and Prives C: Transcriptional regulation by p53: One protein, many possibilities. Cell Death Differ 13: 951-961, 2006.

21. Ma Y, Yang Y, Wang F, Wei Q and Qin H: Hippo-YAP signaling pathway: A new paradigm for cancer therapy. Int J Cancer 137: 2275-2286, 2015.

22. Piccolo S, Dupont S and Cordenonsi M: The biology of YAP/TAZ: Hippo signaling and beyond. Physiol Rev 94: 1287-1312, 2014.

23. Barron DA and Kagey JD: The role of the Hippo pathway in human disease and tumorigenesis. Clin Transl Med 3: 25, 2014. 